\title{
An Experimental Study on Lipid Metabolism in Rats with Obstructive Jaundice ; Focusing on Cholesterol Synthesis
}

\author{
Toshio Masuda, Shunichi Shiozawa and Kenji Ogawa \\ Department of Surgery, Tokyo Women's Medical University Medical Center East
}

\begin{abstract}
Aim : The Aim of this experimental study was to investigate how lipid metabolism is altered in a rat obstructive jaundice model, focusing on cholesterol synthesis.

Materials and methods : A rat obstructive jaundice model was prepared by bile duct ligation. Rats were sacrificed 1,2,3, and 4 weeks after bile duct ligation, and blood was collected, and the total liver was isolated. Normal control rats were also sacrificed, and samples were collected in the same manner. The profile of changes lipid metabolism was evaluated over time.
\end{abstract}

Results and discussion : 1) Serum T-Chol level markedly increased at week 1 . It subsequently declined, while being higher than the control level until week 4. Serum HDL-C level increased until week 2, and concurrently HMG-CoA reductase activity was elevated. These findings indicate increased hepatic cholesterol synthesis during the early stage of obstructive jaundice. 2) Serum HDL-C level, HMG-CoA reductase activity and LR m-RNA expression level all decreased during the period of complete obstructive jaundice at week 4. At this stage, impaired lipid metabolism and depressed hepatic cholesterol synthesis were observed, and these may be referred to liver disorder due to obstructive jaundice.

Conclusion : Thus, lipid metabolism varied in degree of impairment with different stages of obstructive jaundice. Clinically, in the surgery for patients with

Received: July 25, 2011/Accepted: September 15, 2011

Correspondence to: Kenji Ogawa

Department of Surgery, Tokyo Women's Medical University Medical Center East

Nishiogu 2-1-10, Arakawa-ku, Tokyo 116-8567, Japan obstructive jaundice, it is important to take into account such degrees of impairment in lipid metabolism.

Key words: lipid metabolism, cholesterol synthesis, obstructive jaundice

\section{Introduction}

Hepatobiliary disease with obstructive jaundice is often complicated by liver dysfunction, where impairment of excretion of phospholipids, bile acids and cholesterol leads to elevation of serum cholesterol level. The liver takes part in about two-thirds of lipid metabolism in the body and undertakes $75 \%$ of the synthesis of cholesterol which usually amounts to approximately $650 \mathrm{mg}$ per day ${ }^{1)}$. Furthermore, the liver has many regulatory mechanisms involved in cholesterol synthesis and catabolism and plays a role in maintaining constant cholesterol levels in cells and blood ${ }^{2}$.

However, few studies have investigated lipid metabolism, particularly cholesterol synthesis in the liver in the specific condition of obstructive jaundice.

In this study, we prepared a rat obstructive jaundice model and investigated changes in lipid metabolism in rats with obstructive jaundice, focusing on cholesterol synthesis.

\section{Materials and Methods \\ Animals}

Male Wistar rats aged 10 weeks (250 to $300 \mathrm{~g}$ ) were used in the experiment. All animal experiments were conducted in accordance with the Guidelines for Animal Experiment by the Ethical Review Committee of Tokyo Women's Medical University. 


\section{Experimental models}

Preparation of a rat obstructive jaundice model : The rat's abdomen was opened under anesthesia with intraperitoneal Nembutal at $40 \mathrm{mg} / \mathrm{kg}$. The common bile duct was mobilized, and was ligated at two different sites more than $1 \mathrm{~cm}$ apart, followed by resection of the bile duct segment between the two ligated sites. The abdomen was closed by sutures, and an obstructive jaundice model was obtained.

\section{Collection of venous blood and liver tissue}

Rats were sacrificed by intraperitoneal Nembutal overdosage at 1,2, 3, and 4 weeks after bile duct ligation ( $\mathrm{n}=5$ at each week). Blood was collected from the inferior vena cava. The total liver was isolated and weighed, and part of the resected liver tissue was stored frozen.

Normal rats to serve as a control $(n=5)$ were also sacrificed, blood was collected, and the whole liver was resected to obtain liver tissue specimens.

These blood and liver specimens were collected between 9:00 a.m. and 11:00 a.m. in consideration of the circadian rhythm ${ }^{3}$, and the following parameters were measured.

\section{Biochemical analysis}

Serum separated from venous blood was assayed for total bilirubin (T-Bil) as a parameter of jaundice, total cholesterol (T-Chol) and lipoprotein, i.e., low-density lipoprotein-cholesterol (LDL-C), very low-density lipoprotein-cholesterol (VLDL-C) and high-density lipoprotein-cholesterol (HDL-C) as markers related to lipid metabolism.

Using the frozen liver specimens, LDL receptor (LR) m-RNA expression level that expresses capacity of cholesterol uptake ${ }^{2)}$ and 3-hydroxy-3-methylglutaryl- coenzyme A (HMG-CoA) reductase activity, which reflects the capacity of cholesterol synthesis ${ }^{4)}$, were determined.

LR m-RNA expression level was measured by real-time RT-PCR assay according to the procedures described by previous reports ${ }^{5-7)}$. Total RNA extraction from the frozen liver specimens and cDNA synthesis were performed according to the manufacture's instruction (total RNA RNeasy kits, QIAGEN K.K., Tokyo and RT-PCR Kit, invtrogen Japan, Tokyo). A Taq Man primer probe specific to the LR was designed. This primer was hybridized to perform PCR, and the LR m-RNA expression level was measured using SDS7700 (Applied Biosystems Japan Ltd., Tokyo).
HMG-CoA reductase activity was measured using a modification (two-fold scaled up) of the procedures of Kuroda and Endo ${ }^{8}$. The microsomes in the frozen liver specimens were extracted and incubated with a reaction mixture containing $\left[{ }^{14} \mathrm{C}\right]$ HMG-CoA. $\left[{ }^{14} \mathrm{C}\right]$ mevalonolactone was separated from $\left[{ }^{14} \mathrm{C}\right] \mathrm{HMG}$ $\mathrm{CoA}$ in accordance with the procedures described by Nagata et al.9), and HMG-CoA reductase activity was assayed using a liquid scintillation counter LS 6000 SC type (Beckman Coulter Inc., California).

\section{Statistical analysis}

The results are presented as mean \pm SD. Wilcoxson test and Mann-Whitney U test were used for statistical analysis. A difference was considered to be statistically significant when $p<0.05$.

\section{Results}

\section{Obstructive jaundice progression}

\section{T-Bil}

Compared to $0.1 \pm 0.1(\mathrm{mg} / \mathrm{dL})$ in the control, serum T-Bil level increased significantly to at week 1 after onset of bile duct obstruction $(8.8 \pm 0.6, p<0.0001$ in comparison to the control) and at week 2 (17.2 \pm 1.7 , $p<0.0001$ in comparison to that at week 1), indicating development and progression of jaundice. Serum T-Bil level remained unchanged thereafter (17.7 \pm 0.6 at week 3 and $16.6 \pm 1.1$ at week 4 ), and these levels were still significantly higher as compared to the level at week $1(p<0.0001$ in comparison to that at week 1, respectively) (Fig. 1 ).

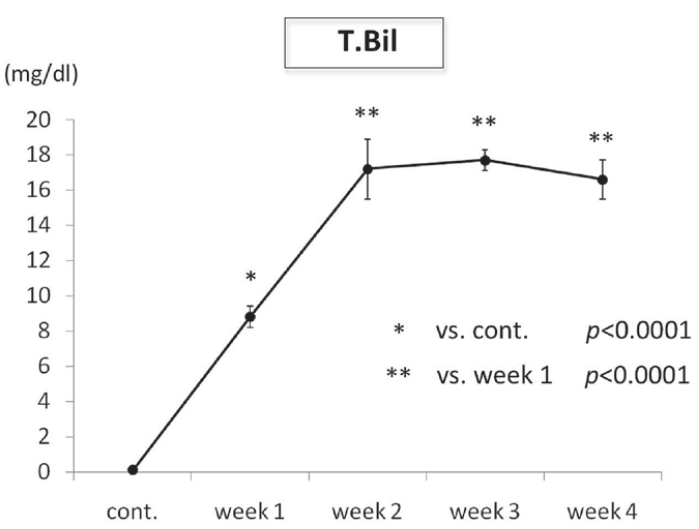

Fig. 1 Compared to the control, serum T-Bil level significantly increased at week 1 after bile duct obstruction, and at week 2 in comparison to that at week 1. It remained unchanged thereafter, and was still significantly higher as compared to the level at week 1 . 
Lipid metabolism in rats with obstructive jaundice

Profile of lipid metabolism with obstructive jaundice progression

\section{1) T-Chol}

Serum T-Chol level $(\mathrm{mg} / \mathrm{dL})$ markedly increased to $160.8 \pm 11.8$ at week 1 as compared to $65.0 \pm 4.6$ in the control. Subsequently it declined steadily to $127.2 \pm$ 6.1 at week $2,104.0 \pm 3.7$ at week $3,85.7 \pm 4.5$ at week 4 , and all the week-1 to 4 levels were still significantly higher than the control level $(\phi<0.0001$, respectively) (Fig. 2 ).

\section{2) LDL-C}

Serum LDL-C level (mg/dL) increased progressively with advancing jaundice from $8.4 \pm 1.5$ in the control to $4.6 \pm 2.2$ at week $1,22.3 \pm 2.1$ at week $2,25.2 \pm 2.5$ at week 3 , and $29.3 \pm 2.2$ at week $4(p<0.0001$ for week1 to 4 levels in comparison to the control, respectively) (Fig. 3 ).

\section{3) VLDL-C}

As seen in serum T-Chol level, serum VLDL-C level $(\mathrm{mg} / \mathrm{dL})$ markedly increased to $91.6 \pm 11.2$ at week 1 as compared to $28.0 \pm 3.0$ in the control and then gradually decreased to $61.0 \pm 8.2$ at week $2,52.8 \pm 6.1$ at week 3 and $39.0 \pm 8.8$ at week 4 , all of which were significantly higher than that in the control $(p<$ 0.0001 for week-1 to 3 levels and $p<0.0008$ for week4 level in comparison to the control) (Fig. 3 ).

\section{4) HDL-C}

Like serum T-Chol and VLDL-C levels, serum HDL-C level $(\mathrm{mg} / \mathrm{dL})$ displayed an increased at week $1(54.6$ $\pm 3.8)$ as compared to the control level $(28.6 \pm 3.4)$,

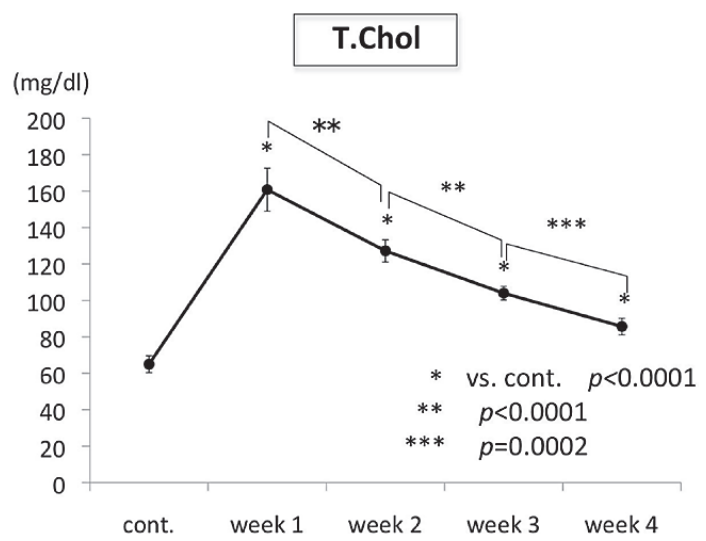

Fig. 2 Serum T-Chol level markedly increased at week 1 as compared to the control. Subsequently it declined steadily. All the week-1 to 4 levels were still significantly higher than the control level.

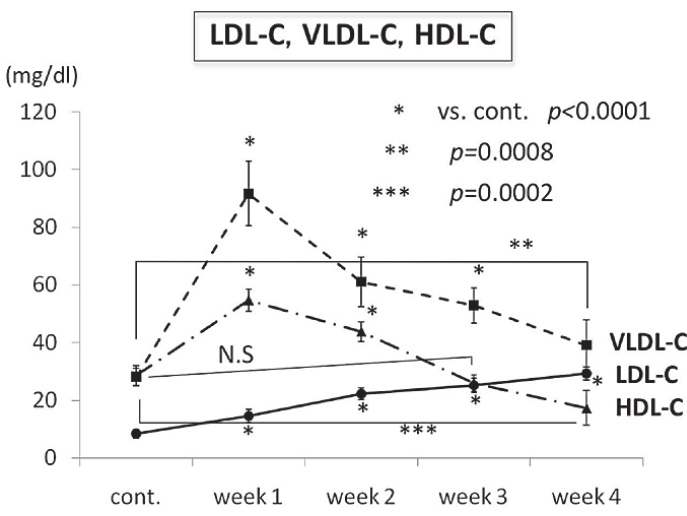

Fig. 3 Serum LDL-C level increased progressively, all the week-1 to 4 levels were significantly higher than the control. Serum VLDL-C level markedly increased at week 1 , and then gradually decreased as seen in serum T-Chol level. All the week-1 to 4 levels were also significantly higher than the control. Serum HDL-C level displayed an increase at week 1 as compared to the control, followed by a gradual decline. The levels at weeks 1 and 2 were significantly higher than the control, but the level was comparable with the control at week 3 , and significantly lower than the control at week 4.

followed by a gradual decline to $43.8 \pm 3.4$ at week 2 , $26.0 \pm 2.9$ at week 3 and $17.3 \pm 6.0$ at week 4 . The HDL-C levels at weeks 1 and 2 were significantly higher than the control level $(\phi<0.0001$, respectively) but the level was comparable with the control at week 3 and significantly lower than the control at week 4 ( $p<0.0002$ ) (Fig. 3 ).

5) LR m-RNA expression level in the liver tissue LR m-RNA expression level $\left(\times 10^{3} / \mathrm{copy} / \mathrm{mL}\right)$ sharply decreased from $244.0 \pm 54.2$ in the control to 36.8 \pm 13.3 at week 1 and continued to decrease significantly thereafter with advancing jaundice, i.e., $7.1 \pm$ 3.3 at week $2,3.4 \pm 1.7$ at week 3 and $3.1 \pm 0.7$ at week 4 ( $\phi<0.0001$, respectively) (Fig. 4 ).

6) $\mathrm{HMG}-\mathrm{CoA}$ reductase activity in the liver tissue HMG-CoA reductase activity $(\mathrm{pmol} / \mathrm{mg} / \mathrm{min}$., measured at $37^{\circ} \mathrm{C}$ ) increased significantly from $23.9 \pm 3.0$ in the control to $39.7 \pm 6.4$ at week 1 and then declined to $30.8 \pm 3.7$ at week $2(\phi<0.0001$ for week-1 to 2 levels in comparison to the control). As was the case with the HDL-C level, the level further de- 


\section{LR m-RNA expression level of liver tissue}

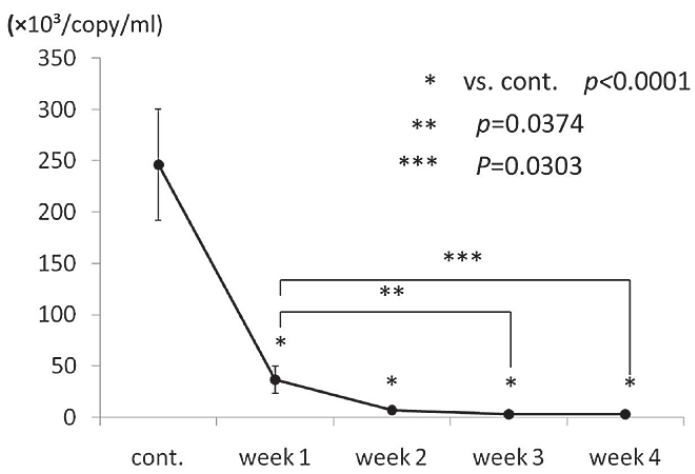

Fig. 4 LR m-RNA expression level sharply decreased at week 1, and continued to decrease significantly thereafter with advancing jaundice.

\section{HMG-CoA reductase activity of liver tissue}

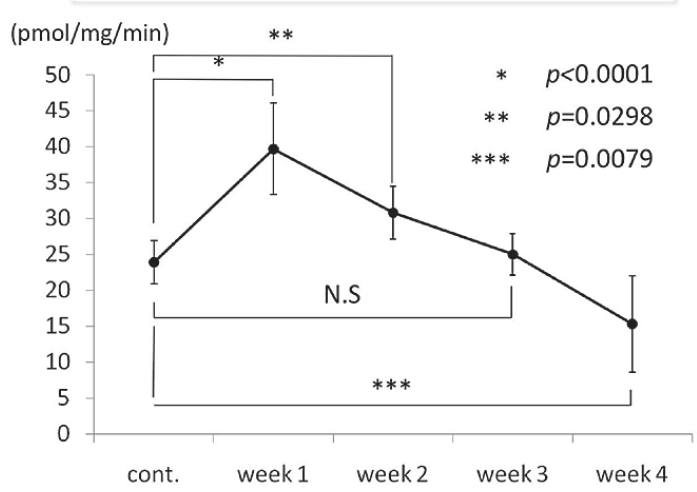

Fig. 5 HMG-CoA reductase activity increased at week 1 as compared to the control, and then declined. week 1 to 2 levels were significantly higher than the control, but as was the case with the HDL-C level, the level at week 3 was comparable with the control, and at week 4 was significantly lower than the control.

creased at week 3 to $25.0 \pm 2.9$, which was comparable with the control, and at week 4 to $15.3 \pm 6.7$, which was significantly lower than the control $(p=$ 0.0079) (Fig. 5 ).

\section{Discussion}

In this study, we investigate how lipid metabolism change over time, what types of disorders develop and how hepatic cholesterol synthesis proceeds in the presence of advancing jaundice.
Hepatic cholesterol synthesis is broadly classified into endogenous mevalonate pathway involving de novo synthesis from acetyl-CoA through mevalonate $^{10,11)}$ and exogenous receptor-mediated pathway from LR-mediated uptake of the serum $\mathrm{LDL}^{12)}$. In the former pathway, HMG-CoA reductase, a microsomal enzyme, is considered to catalyze the ratelimiting step of cholesterol synthesis. The latter mainly takes part in the catabolism of cholesterol via LR on the cell surface. Tissue cholesterol and serum cholesterol are delivered successively to such lipoproteins as VLDL and HDL, and ultimately, metabolized to LDL which binds with LR. After binding with the receptor, the LDL is taken into hepatic cells by endocytosis.

In the present study, we measured the HMG CoA reductase activity and LR m-RNA expression level in the liver tissue, which served as indicators of the capacity of cholesterol synthesis ${ }^{4)}$ and that of cholesterol uptake $\mathrm{e}^{2)}$ in the liver, respectively. In general, a feedback mechanism exists between the LR expression and HMG-CoA reductase activity, and hepatic cholesterol synthesis is controlled by both of them ${ }^{13)}$. Thereby, hepatic cholesterol content and serum cholesterol level remain constant to maintain cholesterol homeostasis. In recent years, the regulation mechanism of cholesterol synthesis via involvement of HMG-CoA synthase in addition to LR and HMG-CoA reductase has been elucidated at the genetic lev$\mathrm{el}^{14-16)}$

Regarding obstructive jaundice progression, the present data demonstrate that serum T-Bil level increased until 2 weeks after the onset of bile duct obstruction and reached a plateau at 3 weeks. This finding is similar to the experimental results reported by Shirakawa ${ }^{17)}$. This is also in accordance with the fact that in the clinical setting serum T-Bil level rises to a certain level at which it reaches a plateau in patients with obstructive jaundice.

As for lipid metabolism, serum T-Chol level was found to have risen sharply at 1 week of obstruction, namely, at the early stage of obstructive jaundice. This serum level then declined gradually, yet continued to be higher than the control level until week 4. HDL-C, which delivers tissue cholesterol to the liver (reverse cholesterol transport), was noted to increase until 2 weeks of obstruction. Concurrently, HMG-CoA reductase activity also increased, reflecting enhanced hepatic cholesterol synthesis with a 
consequent elevation of the excretory form of cholesterol from the liver, VLDL, and its metabolite, LDL. There was a marked decrease in LR m-RNA expression level following the onset of obstruction, indicating a down-regulation through the feedback mechanism with the aforementioned HMG-CoA reductase activity. Obstructive jaundice is associated with impediment of bile juice secretion, leading to inhibition of the enterohepatic circulation of bile acids which represents the most important excretory pathway of cholesterol, and the resultant accumulation of cholesterol in the blood. It is considered that cholesterol excretory disorder, increased hepatic cholesterol synthesis, transient enhancement of reverse cholesterol transport from peripheral tissues and depressed mechanism of cholesterol catabolism by $L R$ are implicated in an intricate manner in the marked T-Chol elevation seen at 1 week, i.e., early after the onset of obstructive jaundice.

Histologically, regeneration of hepatic cells and proliferation of the bile duct are noted in patients with obstructive jaundice ${ }^{18}$. In general, the synthesis of cholesterol and phospholipids required for the formation of cellular membranes are stimulated in liver regeneration ${ }^{13,19)}$. In other words, a large quantity of cholesterol is required for liver regeneration, so that HMG-CoA reductase activity increases to fulfill the need with a consequent increase of cholesterol synthesis ${ }^{7}$. The present data at 1 week of obstruction exactly coincide with this fact. From the viewpoint of lipid metabolism, the present results also provide evidence in support of liver regeneration occurring during the early stage of obstructive jaundice.

Most patients with obstructive jaundice we clinically encounter have a complete jaundice state that is characterized by elevated T-Chol and lowered HDL-C level in terms of serum lipids ${ }^{20)}$. The present data at week 4 of choledochal obstruction correspond to complete obstruction as shown by a slight decrease in T-Chol level though remaining higher than the control level together with lower HDL-C level as compared to that in the control. Furthermore, both LR m-RNA expression level and HMG-CoA reductase activity were depressed, suggesting failure in the underlying feedback mechanism, impaired lipid metabolism and absence of liver regeneration. The condition may be referred to as liver disorder due to prolonged obstructive jaundice.
Several studies have demonstrated the clinical usefulness of serum HDL-C level as an indicator of liver function after hepatectomy or after biliary drainage for the treatment of obstructive jaundice ${ }^{21,22)}$. The present data also, show the elevation of HDL-C level at 1 week of obstruction, which remained high during the period with increased cholesterol synthesis and higher liver regeneration capacity, followed by a decrease at week 4 , when hepatic regeneration was very unlikely due to depressed hepatic cholesterol synthesis.

Many recent clinical reports have stated that preoperative biliary drainage is unnecessary for lower bile duct obstruction in cancer patients with obstructive jaundice even if a pancreatoduodenectomy is performed ${ }^{23-28}$. On the other hand, there is controversy over the need ${ }^{29)}$ or lack of need ${ }^{30)}$ for preoperative biliary drainage for upper bile duct obstruction, such as hilar bile duct carcinoma, because a highly invasive major hepatectomy is indicated in such cases. The present data suggest that from the viewpoint of lipid metabolism, biliary drainage does not necessarily need during the early stage of obstructive jaundice with high liver regeneration capacity, namely, with elevated serum HDL-C level and highly invasive hepatectomy is feasible. We also demonstrated the recovery of HDL-C level after biliary drainage even in patients with jaundiced liver ${ }^{31)}$. In patients with complete obstructive jaundice with depressed HDL-C level, it may be of advantage to suspend a hepatectomy until HDL-C level is elevated via sufficient reduction of jaundice by biliary drainage to achieve recovery of impaired lipid metabolism and liver regeneration capacity.

\section{Conclusion}

We obtained the following findings regarding changes in lipid metabolism in a rat obstructive jaundice model with primary reference to cholesterol synthesis.

1) Serum T-Bil level increased until 2 weeks of bile duct ligation, reached a plateau at week 3 , and remained at this level thereafter. 2) Serum T-Chol level markedly increased at week 1 , namely at the early stage of obstructive jaundice. It subsequently declined, while being higher than the control level until week 4. Serum HDL-C level increased until week 2 , and concurrently HMG-CoA reductase activity was elevated. Elevations of VLDL and LDL lev- 
els were also observed. These findings reflect increased hepatic cholesterol synthesis during the early stage of obstructive jaundice. 3) Serum HDL-C level, HMG-CoA reductase activity and LR m-RNA expression level all decreased during the period of completion of obstructive jaundice at week 4 . The rats at this stage with impaired lipid metabolism and depressed hepatic cholesterol synthesis were in a condition that may be referred to as liver disorder due to obstructive jaundice.

Thus, lipid metabolism varied in degree of impairment with different stages of obstructive jaundice. In particular, cholesterol synthesis relating to liver regeneration was impaired at the stage of complete obstructive jaundice. Clinically, in the surgery for cancer patients with obstructive jaundice, it is important to determine the need for preoperative biliary drainage and operative procedures while taking into account such degrees of impairment in lipid metabolism.

\section{References}

1) Grundy SM, Mok HY : Determination of cholesterol absorption in man by intestinal perfusion. J Lipid Res 18 : 263-271, 1977

2) Brown MS, Goldstein JL : How LDL receptors influence cholesterol and atherosclerosis. Sci Am 241: 52-60, 1984

3) Balasubramaniam S, Szanto A, Roach PD : Circadian rhythm in hepatic low-density- lipoprotein (LDL) receptor expression and plasma LDL levels. Biochim J 298 (Pt1) : 39-43, 1994

4) Trentalance A, Leoni S, Mangiantini MT, et al : Regulation of 3-hydroxy-3-methylglutaryl coenzymeA reductase and cholesterol synthesis and esterification during the first cell cycle of liver regeneration. Biochim Biophys Acta 794 : 142-151, 1984

5) Lee LY, Mohler WA, Schafer BL, et al : Nucleotide sequence of the rat low density lipoprotein receptor cDNA. Nucleic Acids Res $17: 1259-1260,1989$

6) Reinholz MM, Iturria SJ, Ingle JN, et al : Differential gene expressin of TGF-beta family members and osteopontin in breast tumor tissue : analysis by realtime quantitative PCR. Breast Cancer Res Treat 70 : $255^{-269,} 2002$

7) Takahashi H, Shiozawa S, Tsuchiya A, et al : Experimental studies on liver regeneration after partial hepatectomy in rats with obstructive jaundice followed by biliary drainage; with special reference to lipid metabolism. J Tokyo Women's Med Univ 75 : 115-123, 2005

8) Kuroda M, Endo A : Inhibition of in vitro cholesterol synthesis by fatty acids. Biochim Biophys Acta 486 : 70-81, 1977

9) Nagata $Y$, Hidaka $Y$, Isida F, et al : Effect of simvastatin (MK-733) on the regulation of cholesterol synte- sis in HepG2 cells. Biochem Pharmacol 40 : 843-850, 1990

10) Lichtenstein $\mathrm{AH}$ : Intestinal cholesterol metabolism. Ann Med Fev $22:$ 49-52, 1990

11) Goldstein JL, Brown MS : Regulation of the mevalonate pathway. Nature $341: 425-430,1990$

12) Brown MS, Goldstein JL : A Receptor-Mediated Pathway for Cholesterol Homeostasis. Science $232: 34-$ 47, 1986

13) Takeuchi N, Katayama Y, Matsumiya K, et al : Feedback control of cholesterol synthesis in partially hepatectomized rats. Biochim Biophys Acta 450 : 57-68, 1976

14) Yokoyama C, Wang X, Briggs MR, et al : SREBP-1, a basic-helix-loop-helix-leucine zipper protein that controls transcription of the LDL receptor gene. Cell $75: 187-197,1993$

15) Wang X, Sato R, Brown MS, et al : SREBP-1, a membrane-bound transcription factor released by sterolregulated proteolysis. Cell $77: 53-62,1994$

16) Osborn TF, Bennett M, Rhee $K$ : Red 25, a protein that binds specifically to the sterol regulatory region in the promoter for 3-hydroxy-3-methyl-glutaryl-coenzyme-A reductase. J Biol Chem 267 : 1897318982, 1992

17) Shirakawa $M$ : Biochemical studies on the time limit for surgical release of obstructive jaundice after common bile duct ligation in rats and dogs. Jpn J Gastroenterol Surg $11: 359-368,1978$

18) Trams EG, Symeonidis A : Morphologic and functional changes in the livers of rats after ligation or excision of the common bile duct. Am J Pathol 33 : 13-27, 1956

19) Field FJ, Mathur SN, Labrecque DR : Cholesterol metabolism in regenerating liver of the rat. Am J Physiol 249 : 679-684, 1985

20) Minemura M, Watanabe A : Regulation of fatty metabolism in the liver. Nippon Rinsyo $59: 436-441$, 2001

21) Katsuramaki T, Hirano K, Mukaiya M, et al : Changes in lipid metabolism after hepatectomy and usefulness of HDL-cholesterol as an indicator of hepatic function. J Jpn Soc Parenteral \& Enteral Nutrition $11: 212-215,1996$

22) Kumazawa K, Haga S, Hosokawa T, et al : Evaluation of the significance of reducing jaundice by PTCD in terms of changes in liver function. Jpn J Gastroenterol Surg $28: 778-785,1995$

23) Povosky SP, Karpeh MS, Conlon KC, et al : Association of preoperative biliary drainage with postoperative outcome following pancreatoduodenectomy. Ann Surg 230 : 131-142, 1999

24) Sewnath ME, Birjmohun RS, Rauws EA, et al : The effect of preoperative biliary drainage on postoperative complications after pancreatoduodenectomy. J Am Coll Surg 192 : 726-734, 2001

25) Martignoni ME, Wagner M, Krahenbuhl L, et al : Effect of preoperative biliary drainage on surgical outcome after pancreatoduodenectomy. Am J Surg 181 : 52-59, 2001

26) Pisters PW, Hudec WA, Hess KR, et al : Effect of preoperative biliary decompression on pancreatoduo- 
Lipid metabolism in rats with obstructive jaundice

denectomy-Associated morbidity in 300 consective patients. Ann Surg $234: 47-55,2001$

27) Miura O, Hanyu F, Imaizumi T, et al : Advantages and disadvantages of preoperative percutaneous transhepatic biliary drainage for obstructive jaundice. Biliary tract \& Pancreas $10: 561-567,1989$

28) Takada $T$ : Is preoperative biliary drainage necessary according to evidence-based medicine? J Hepatobiliary Pancreat Surg $8: 58-64,2001$

29) Nagino M, Kamiya J, Uesaka K, et al : Complications of Hilar Cholangiocarcinoma. World J Surg 25 : 1277-1283, 2001

30) Cherqui D, Benoist S, Malassagne B, et al : Major liver resection for carcinoma in jaundiced patients without preoperative biliary drainage. Arch Surg $135: 302-308,2000$

31) Tsuchiya A, Haga S, Watanabe O, et al : Experimental study on lipid and bilirubin metabolism after biliary drainage for obstructive jaundice. J Surg Res $96: 50-55,2001$ 